Radio Interferometry: Theory, Techniques and Applications,

\title{
THE NRAO MILLIMETER ARRAY SITE SURVEY
}

FRAZER N. OWEN

NRAO, P. O. Box O, Socorro, NM 87801

ABSTRACT The measurements being made to pick a site for the NRAO Millimeter Array are described. The instruments, techniques and preliminary results are discussed, along with the strategy for picking the final site for the array.

\section{INTRODUCTION}

The NRAO Millimeter Array (MMA) is currently a proposal being reviewed by the National Science Foundation of the United States. The proposal asked for funds to build a 40 element synthesis array of movable $8 \mathrm{~m}$ antennas. The array would operate between 30 and $350 \mathrm{GHz}$ with its prime band being the 200 to $300 \mathrm{GHz}$ atmospheric window. In this window resolution of 0 .' 1 is required and seems feasible. This resolution implies an array roughly $2 \mathrm{~km} \mathrm{EW}$ by $3 \mathrm{~km} \mathrm{NS}$, the larger extent NS allowing observations of southern objects without major distortion of the synthesized beam.

The combination of high frequencies and high resolution implies a high site which is both dry and large. For this reason we restricted our search for a site to elevations above $9000 \mathrm{ft}(2800 \mathrm{~m})$ which have enough flat ground to build a large, movable array. We have restricted our most detailed studies to sites south of latitude $36^{\circ} \mathrm{N}$ on U.S. territory. The latitute restriction results from a desire to avoid the more severe winter weather patterns in the southwestern U.S. and to maximize the fraction of the southern sky available for study. However, several promising sites north of this limit have been investigated. Also sites outside U.S. territory have been considered but all of these have been ruled out for cost and logistical reasons.

Within the limitiations described we have currently reduced our list of potential sites to three. Two of the sites are in the White mountains of eastern Arizona, one near Springerville, AZ at an elevation of $9200 \mathrm{ft}(2900 \mathrm{~m})$ and one near Alpine, $\mathrm{AZ}$ at $9800 \mathrm{ft}(3100 \mathrm{~m})$. The third site is in the Magdelena mountains near Socorro, NM at $10,600 \mathrm{ft}(3400 \mathrm{~m})$. We are currently testing two of these sites: the Springerville and the Magdelena mountains sites. We are also testing Mauna Kea in Hawaii as a fiducial "best" site and to help in the SAO evaluation of that site for their proposed submillimeter array. Unfortunately Mauna Kea does not have a large enough, flat area for the array so is not being considered as a potential site. 
The main site testing tools for the MMA are three 225-GHz tipping radiometers. The concept of a standalone tipping radiometer at millimeter wavelengths for measuring atmospheric opacity was first developed and a prototype built by John Payne in Tucson, Arizona. Initial measurements were made on the VLA site with it in 1984. The current version of the "tipper" was engineered by Sandy Weinreb and built in the NRAO Central Developement Laboratory in Charlottesville, Virginia. With the help of many of the VLA engineers, most notably Paul Lilie, and numerous students, with Mark McKinnon playing the most important role, we have been taking data since 1986 with the tippers. Since 1988 Gerry Petencin has been the lead engineer working on the project and he has made many modifications and improvements to the instruments. Each tipper consists of an insulated box about one cubic foot in volume filled with electronics and an external mirror, controlled by a stepping motor, which feeds the signals from various different elevations into the box. A chopper wheel in the box alternately directs the signals from the sky or one of two fixed temperature loads, $45 \mathrm{C}$ and $65 \mathrm{C}$, into the signal path. From the two internal loads both the zero point and the gain of the radiometer are continuously calculated producing a very stable system.

One of these tippers has been operating on the site in the Magdelena mountains since November of 1986. A second tipper has operated at the Caltech Submillimeter Observatory on Mauna Kea since August, 1989. The third tipper started making measurements on the Springerville site in September of 1990. The Springerville site has no power or phone communications. To deal with this situation we are running the Springerville tipper on solar and wind power. We have also installed a radio telephone link to a Forest Service fire tower about $30 \mathrm{~km}$ away.

Two principle types of measurements are made with the tippers: tipping scans and stability scans. A tipping scan consists of a measurement of the sky brightness at $225 \mathrm{GHz}$ at 11 different elevations. A model for the zenith opacity is then fitted to each scan. One tipping scan is made every ten minutes. So far in the project we have measured about 150,000 opacities.

The stability scan consist of the measurement of a one hour time series of the zenith sky temperature. One is carried out every five hours. When the opacity is $<<1$ and the sky is free of clouds with water droplets, the sky temperature should be proportional to the electrical path length due to water vapor. Thus from the time series we can estimate the phase fluctuations seen by an individual antenna. We parameterize each scan by calculating the Allen varience over nine different timescales varying by factors of 2 from 3.6 seconds to 15 minutes. We then scale the results to the equivalent $\mathrm{rms}$ for relation to phase fluctuations. Estimates of the instrumental contribution are made regularly (every three to seven observations) by pointing the mirror down at a temperature stable absorber instead of up at the sky. Corrections are made to the data from long term averages of the calibration scans. Combining these numbers with a velocity for the water vapor clouds, we can estimate the variation in interferometer phase due to water vapor which we believe is the major source of millimeter seeing variations. When large sky temperature flucuations occur due to water droplets or when the opacity is very high, useful 
millimeter astronomical observations are not possible anyway. Thus from a combination of the two types of scans we can get a estimate of the useable time on a site for millimeter interferometry.

In addition to opacity and stability scans, we measure ambient temperature on each site and wind velocity and direction on the Magdelena and Springerville sites every ten minutes to complete our site profile.

\section{PRELIMINARY RESULTS}

Up until the current time most of our efforts in this project have gone into getting the tippers working and setting up the computer controls, communications and other logistics which go with running a multisite project. The tippers are really complete, miniature radio telescopes with all the components and potential problems associated with their larger cousins. Now the data from each of the three systems is being collected daily in Socorro and we have begun trying to digest the results.

During September and October 1990, data have been taken on both the Springerville and Madelenas sites. However, the dry weather in the southwestern U.S. is just beginning and too little data have been taken to draw any significant conclusions. However, several initial conclusions can be drawn in the comparision between the Mauna Kea and Magdelenas sites. First, as expected, the Mauna Kea site is clearly better year round than the Magdelenas. The average conditions in the best six months in the Magdelenas are similar to the year round average for Mauna Kea. Also the lowest opacities seen on both sites are similar, about 0.03 at $225 \mathrm{GHz}$. However, the worst six months in the Magdelenas are much worse. About three months of the bad period during July, August and September are almost a total loss for 1 millimeter astronomy (but not the lower millimeter frequencies) and the other three months are significantly worse than average conditions on Mauna Kea. Thus, crudely, about twice as much time per year occurs on Mauna Kea at any given opacity below 0.2 at $225 \mathrm{GHz}$.

The stability measurements show that most of the power in the fluctuations under the good conditions described above occurs on timescales longer than one minute. This is what we expected based on a Kolmolorov spectrum for the atmospheric turbulence and a velocity typically of $10 \mathrm{~km}$ $\mathrm{sec}^{-1}$ or less. On both sites we see many periods when coherent observations should possible at one millimeter even on the longest baselines.

For similar ranges of opacity we see similar atmospheric stability on Mauna Kea and in the Magdelenas; however, we have not done a detailed comparison of opacity and stability on either site. On both the Mauna Kea and Magdelena sites we see a diurnal variation in the stability. The effect seems to occur over more of the day on Mauna Kea than on the southwestern sites. On Mauna Kea we see an increase in the fluctuations over the either daylight period and decreasing at night. On the Magdelenas site we observe the biggest fluctuations in the afternoon with the mornings generally being good. However, further study is necessary separating out the good opacity conditions on both sites to be studied separately before any final conclusions are drawn. 
Much analysis remains to be done on existing and future datasets. We plan to study the relationship of combinations of variables on various sites. For example, one might expect the stability to worsen when the wind is blowing hard. We would like to know how closely the opacity correlates with the stability. We also plan to compare our data with the SAO interferometer measurements of the phase from observations of a geostationary satellite.

For our site decision our major strategy is to compare the opacity, stability, wind, and temperature on the Springerville and Magdelenas sites for one year. We hope that from these data and other considerations we can make a site decision in the summer or fall of 1991. If the choice is a close call, we may test the Alpine site as a final piece of information. However, we expect the Alpine site to be only slightly better than the Springerville site since it is only $600 \mathrm{ft}$ higher and about 20 miles distant. Thus we hope to be able to finish our surveys on all three sites in 1991.

Alan Rogers: At these very low opacities have your considered possible contributions to the path length fluctuations which come from the day atmosphere?

Frazer Owen: Yes, a contribution from a dry term to the fluctuations is a possibility. However, initial comparison of the SAO interferometer fluctuations with our sky temperature fluctuation data on Mauna Kea suggests too good a correlation for the dry term to be very important.

Melvyn Wright: Have you looked at the correlation of fluctuations with baseline using two devices on the same mountain?

Frazer Owen: No, we have not done this. We have compared the radiometers with one another on the same mountain top, but only to verify they are measuring the same thing. 